\title{
COMMUNICATIONS
}

\section{CRYOSURGERY IN TREATING RETINAL DETACHMENT AND OTHER EYE DISORDERS* ${ }^{\dagger}$}

\author{
BY \\ HARVEY A. LINCOFF AND JOHN M. MCLEAN \\ From the Department of Surgery (Ophthalmology) New York Hospital-Cornell Medical Center $\ddagger$
}

\section{History}

CRYosurgery for the treatment of retinal detachment was attempted in 1933 by Bietti (1934), and later in the same year by Deutschmann (1933). The former used a mixture of solid carbon dioxide and acetone in the handle of a metal probe and produced chorioretinal lesions similar to those that result from diathermy. The latter achieved a like effect by applying carbon-dioxide snow directly to the sclera. Bietti's (1950) interest in cryosurgery was later renewed in connexion with the treatment of glaucoma, and in 1950 he reported on a cyclocryothermy operation which he found less effective than cyclodiathermy, but of value in inflammatory glaucoma.

Our interest in cryosurgery began in 1962 when we were looking for a substitute for diathermy in the treatment of retinal detachment. We undertook this search because, employing full-thickness scleral buckling procedures, we found the scleral necrosis resulting from full-thickness diathermy to be undesirable beneath a synthetic implant.

In our first experiment in the spring of 1962 we used various types of solid carbondioxide applicators, all of which are limited in temperature to that of carbon dioxide $\left(-79^{\circ} \mathrm{C}\right.$.) minus the amount of cold lost in transfer. The probe temperature varied unpredictably from $-20^{\circ} \mathrm{C}$. to $-50^{\circ} \mathrm{C}$., depending upon the level to which it was filled at the moment of use, and the amount of frost on the metal extension. Because it was not insulated, the instrument tended to stick to the tissues. Most important, it could not continue to supply a sufficient degree of cold to combat the load imposed by the warming effect of the uveal circulation. It became apparent that we needed a system with a circulating refrigerant equivalent in capacity to the uveal circulation.

Many of these problems were solved with the development of the Cooper (1963) Linde Cryosurgical Unit for the treatment of Parkinsonism. This apparatus circulates liquid nitrogen through an insulated probe and delivers a controlled temperature ranging from $+37^{\circ} \mathrm{C}$. to $-180^{\circ} \mathrm{C}$. The frozen tip can be disengaged

\footnotetext{
* Received for publication December 28, 1964

* Received for publication December 28, 1964.

\$ These studies were supported in part by a Fight for Sight Grant from the National Council to Combat Blindness and by a Grant from the National Institute of Neurological Diseases and Blindness. 
by shutting off the nitrogen flow, thus permitting the probe to be warmed by the tissues. One of these machines was made available to us by the Linde Division of the Union Carbide Co. in November, 1962, and was first used in a series of animal experiments in an effort to create a therapeutic lesion for the treatment of retinal detachment.

It became apparent that a smaller and more manœuvrable apparatus was needed for ocular surgery. One was developed at the New York Hospital-Cornell Medical Center, and the first bench-made model was ready for trial in the spring of 1963 (Figs 1 and 2). Like the neurosurgical device, it uses liquid nitrogen as a cooling agent, but this is stored in the handle of the probe because the semi-flexible conduit of



FIG. 1.-Ophthalmic cryosurgery apparatus.

the neurosurgical apparatus is too restrictive for ocular surgery. The stored nitrogen is adsorbed on a cellular matrix so that the instrument can be turned in all attitudes without back-spilling. Sufficient cooling material is stored for an hour's use. The instrument can be refilled in 3 minutes. The temperature of the probe is monitored by a sensing device in its tip and recorded by a direct writer. A servo-mechanism permits the desired temperature to be pre-set on the recorder and then activated by a foot-switch. The instrument is capable of freezing tissue at a rate of temperature change of $20^{\circ} \mathrm{C}$. per second. In the earlier models it was warmed by stopping the flow of nitrogen and allowing the probe to be heated by the circulation in the adjacent tissues. The warming rate was about $3^{\circ} \mathrm{C}$. per second, and thus it took 10 seconds before the instrument could be disengaged from a $-30^{\circ} \mathrm{C}$. application. Because this 


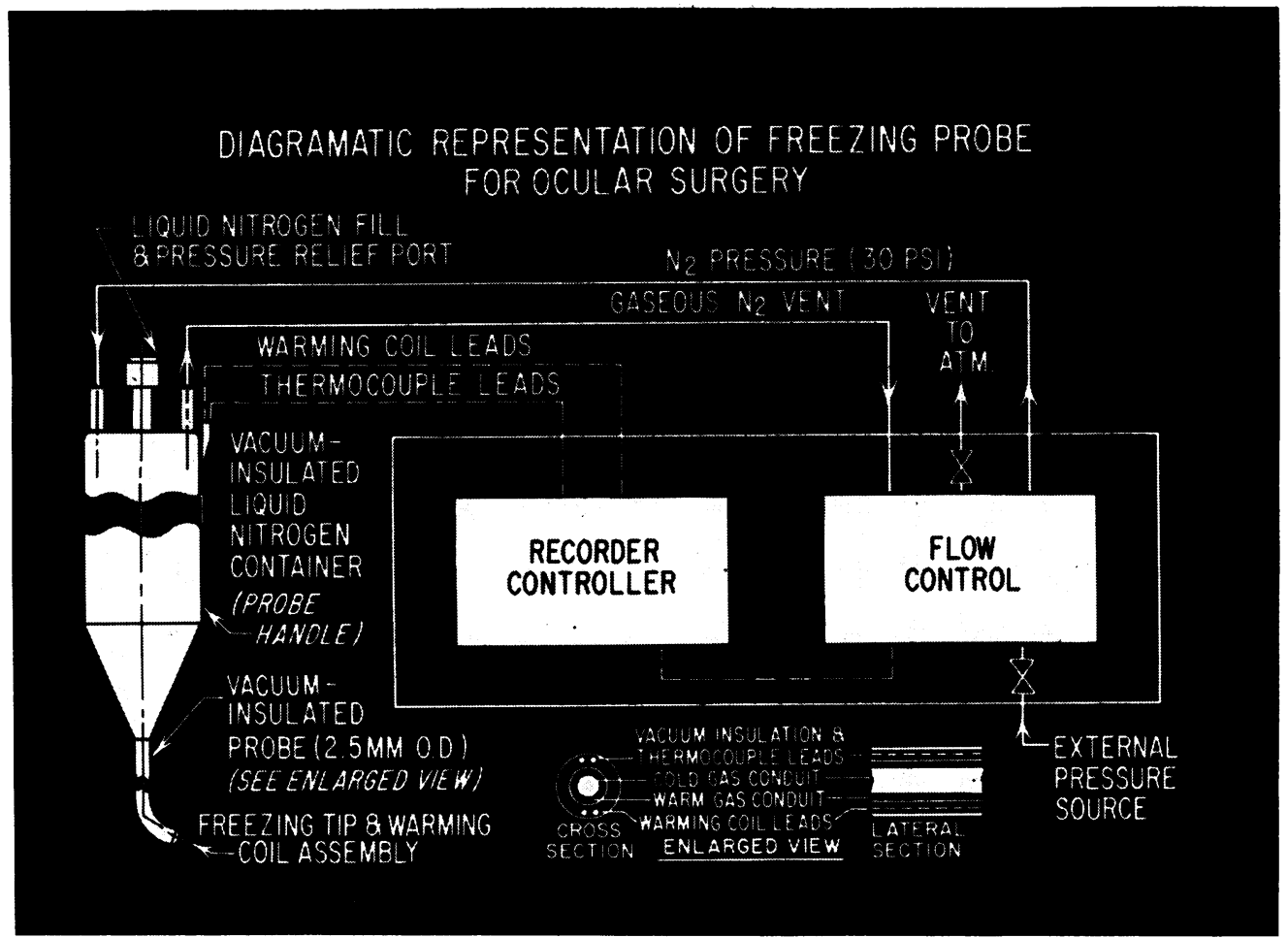

FIG. 2.-Flow diagram of ophthalmic cryosurgical apparatus.

proved inadequate, we incorporated into the system a heating device which restores the end of the probe to body temperature $\left(+37^{\circ} \mathrm{C}\right.$. $)$ at $15^{\circ}$ per second. With the new instrument the application of cold is only slightly more prolonged than that of diathermy.

\section{Therapeutic Possibilities of a Sub-freezing Temperature Probe}

Cryosurgery appears to offer three possibilities in the treatment of eye disorders: (1) At temperatures just below freezing, for example $-10^{\circ} \mathrm{C}$., the tip of the probe adheres firmly to tissue. Advantage is taken of this property to extract cataractous lenses. (2) Temperatures of $-20^{\circ} \mathrm{C}$. to $-50^{\circ} \mathrm{C}$. applied to the wall of the eye cause moderate cellular necrosis in the pigment and neuro-epithelial layers, but the predominant effect is an exudative response at the chorioretinal interface similar to that produced by diathermy. (3) Lower temperatures in the range of $-80^{\circ} \mathrm{C}$. to $-120^{\circ} \mathrm{C}$. can be used if the primary objective is tissue destruction. This temperature range has been used in an effort to diminish ciliary body function for the treatment of glaucoma and to destroy intra-ocular tumours.

\section{Retinal Detachment}

Most of our cryosurgical work has been devoted to the treatment of retinal detachment, and it is in this area that it has been most fruitful. Experimental freezing of animal retinas was begun in 1962 (Lincoff, 1964). Retinal lesions were made with 
the cold probe at various combinations of temperature and time. They were followed clinically for various lengths of time and the animals sacrificed at intervals so that the progression of the lesions could be studied histologically. Our aim was to find an exudative lesion that would yield a firm chorioretinal adhesion with minimal damage to other structures.

\section{Ophthalmoscopic Examination}

An application of $-20^{\circ} \mathrm{C}$. to the sclera of a rabbit produced a white lesion in the retina in a few seconds. When the temperature was raised above $2^{\circ} \mathrm{C}$., the lesion faded and there remained only a faint grey area of oedema. Little change took place over the next five days, but thereafter retinal pigment disorganization became evident at the periphery of the lesion. By the end of ten days the pigmentary change had extended throughout the lesion. The area appeared pale yellow and evenly speckled with pigment. It looked like a favourable diathermy lesion, and when it was examined histologically, revealed a firm chorioretinal adhesion.

\section{Gross Examination}

All animal eyes used in our experiments were fixed in formalin, washed, and passed through 75 per cent. and 95 per cent. alcohol. They were sectioned with a razor blade through the site of the lesion in an anterior-posterior direction. The most conspicuous effect of the cryosurgical procedure was adhesion of the retina in the area of the lesion and absence of scleral change. Elsewhere the retina was nearly always detached as a result of fixation and manipulation (Fig. 3). The phenomenon of chorioretinal adhesion in the presence of artificial detachment appeared as early as the second day.

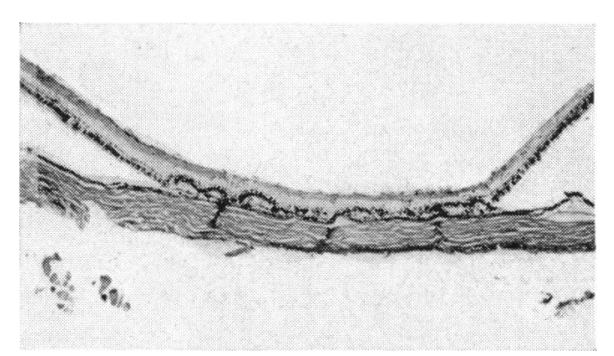

Fig. 3.-Chorioretinal adhesion following cryopexy.

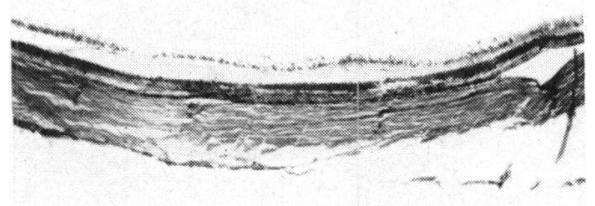

FIG. 4.- Rabbit retina 24 hours after an application of $-20^{\circ} \mathrm{C}$.

\section{Microscopic Examination}

Microscopic examination revealed that the immediate effect of an application of cold is oedema of all layers (Fig. 4). The retina becomes thickened and thrown into folds, the choroid congested. The sclera shows swelling and separation of fibres, and the scleral nuclei lose their horizontal alignment. During the next four days (Fig. 5), oedema and congestion increase and the nuclear layers of the retina become further disorganized. Thereafter, oedema is resolved and choroidal congestion absorbed (Fig. 6). By the seventh day, milder lesions show relatively complete 


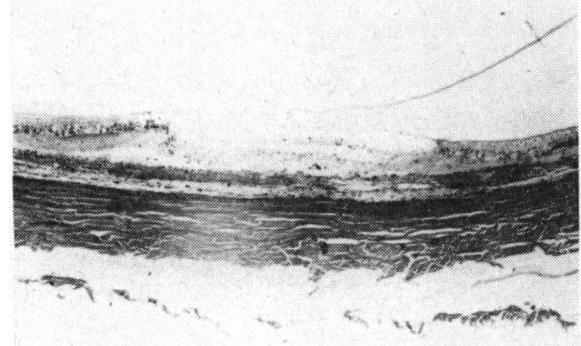

Fig. 5.-Rabbit retina 4 days after an application of $-20^{\circ} \mathrm{C}$.

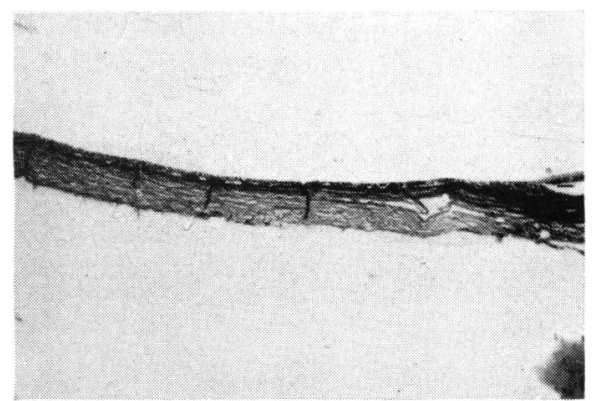

FIG. 6.-Rabbit retina 21 days after an application of $-20^{\circ} \mathrm{C}$.

retraction and firm chorioretinal adhesion. The retina is half its normal thickness. The rods and cones, which are particularly sensitive to cold, are gone. The number of ganglion cells is reduced. What remains of the pigment epithelium is reoriented at the chorioretinal interface. The choriocapillaris is moderately atrophic, and in subsequent months continues to atrophy so that only the larger choroidal vessels survive.

Although freezing affects the nuclei of scleral fibres, it causes no change in the collagenous fibres themselves. The scleral nuclei resume normal alignment at the end of a week. Sections of muscle and tendon that have been frozen first show separation of fibres with oedema and haemorrhage, but by the end of a week these tissues also appear normal. Large vessels, such as vortex veins, suffer no permanent damage when frozen and do not rupture, bleed, or occlude.

The experiments on animal eyes gave clinical histological evidence that subfreezing temperatures could cause a chorioretinal exudative lesion which, when healed, would produce a chorioretinal adhesion. We now needed to determine what therapeutic temperatures were appropriate for the human eye. These were found to be less consistent than in animals, the range varying from $-20^{\circ} \mathrm{C}$. to $-50^{\circ} \mathrm{C}$. applied for 2 to 15 seconds. In practice the temperature is selected for each individual patient in much the same manner as the level of diathermy is chosen. The first few applications that localize the retinal break are monitored with the ophthalmoscope. We have come to know that a minimal whitening of the retina or choroid constitutes an adequate therapeutic lesion. The starting temperature is arbitrarily set at $-40^{\circ} \mathrm{C}$. If the lesion comes through too rapidly, the temperature is raised; if it comes through too slowly, it is lowered. The aim is to find the temperature that will turn the retina white in 3 or 4 seconds. This setting is then used to apply cryopexy to the area of the retinal break. The degree of cold required varies with the thickness and vascularity of the sclera. Anterior lesions require less cold, posterior lesions more. The area of the vortex vein requires $-50^{\circ} \mathrm{C}$., as do the hyperaemic tissues encountered in re-operations.

The human eye tolerates a fairly broad range of cold, so that while it is desirable that lesions be minimal, this is not as critical as with diathermy. Over-freezing in the range of $-20^{\circ} \mathrm{C}$. to $-50^{\circ} \mathrm{C}$. has no effect on the sclera, does not cause choroidal haemorrhage, and will not perforate the retina or harm the vitreous.

To date, 110 patients with detachment problems have been treated with cryosurgery. Of these 31 were treated with prophylactic cryopexy for retinal holes and 
the remaining 79 had manifest detachments. Prophylactic cryopexy can be applied through conjunctiva and tendon without incision. It is of particular value when photocoagulation is contra-indicated because of vitreous haemorrhage or cataractous lens. We also use it when the retinal lesion is so peripheral as to make it difficult to reach with the photocoagulator. We have been employing prophylactic cryopexy over larger areas than we attempted with photocoagulation or diathermy. The accessibility of the periphery to the external route, and the innocuous course following cryopexy have recently made it possible for us to treat $360^{\circ}$ of peripheral degeneration.

It is in the treatment of manifest retinal detachment that cryosurgery reaches its maximum usefulness. At New York Hospital, as at most institutions, we have turned increasingly to scleral buckling with synthetic implants. This method has resulted in a greatly improved prognosis for cases of detachment. There have, however, been some disadvantages. In many cases scleral dissection is required to permit even diathermy at the retinal level. The combination of dissection, diathermy, and a synthetic implant has sometimes resulted in erosion through the sclera over the long term. Because of this we have recently turned to full-thickness buckling procedures, but the scleral necrosis caused by full-thickness diathermy presented difficulties. This was the primary reason for experimenting with cryosurgery. With cryopexy, full-thickness treatment of the sclera ceases to be a problem, because collagenous tissue is immune to cold; thus full-thickness buckling without scleral dissection has become practicable. One of the peripheral advantages has been the reduced incidence of post-operative scleral infections beneath the implant. Animal experiments indicate that the prerequisite for post-operative endophthalmitis following retinal detachment is a combination of diathermy, staphyloccoci, and a synthetic implant. When diathermy is replaced by cryopexy, the sclera remains sufficiently resistant to the organism to overcome it.

Complications with cryopexy have been minimal. The uveitic response to extensive applications (for example, as many as 60 applications over half the circumference of the globe) is less than that of equivalent diathermy. Subchoroidal extravasation is less frequent. Intra-ocular haemorrhage has never occurred. Animal experiments as well as clinical observations indicate that the vasculature is immune to the levels of cold needed in retinal detachment therapy.

Cryopexy has reduced the problems in re-operation, for the scleral wall remains intact and undamaged. There is no unsafe period of soft or necrotic sclera beyond which it is advisable to wait if the first operation is failing. Re-operation is performed upon a leaking area as soon as the inevitability of such surgery becomes evident and at any time in the first post-operative week.

Cryopexy also makes it easier to re-operate on eyes that have re-detached after buckling procedures in which diathermy and scleral dissection have been used. We now slide the original implants out of their beds through a small opening at the end of the scleral pocket, without uncovering the dissection itself. In thin and necrotic areas we are inclined to leave some portion of Tenon's tissue in place rather than to dissect the eye down to its scleral level, as is required for the re-application of diathermy. Cryopexy is then applied over the old resection, with a greatly reduced risk of perforation. 
The 110 cases referred to in this paper were consecutive and unselected; 94 were repaired by the first procedure, 8 required a second, and 5 a third procedure; in 3 cases we were unable to re-attach the retina.

\section{Cataracts}

A number of eye surgeons have removed cataracts with a cold probe. Three years ago Krwawicz $(1961,1963)$ reported on the technique. He used a carbondioxide acetone probe of relatively simple design. In the past year Bellows (1964) and Kelman and Cooper (1963) have also reported on cataract extractions with cold probes. The former uses a carbon-dioxide device, the latter an electrical probe in combination with circulating ice-water. We have employed the technique with the Cornell instrument in 18 cases and have found it entirely satisfactory. A section of slightly more than $180^{\circ}$ is made, so that the cornea, the endothelium of which is vulnerable to freezing, can be lifted well away from the probe, which is applied to the surface of the lens at $-10^{\circ} \mathrm{C}$. As the lens is lifted free of the iris, the temperature is lowered to $-40^{\circ} \mathrm{C}$. to make the lens-probe bond more secure. The lens is delivered by the sliding technique. Because the cold probe would stick to both structures, it cannot be slid under the iris in order to seize the lens capsule at its equator, as with a capsule forceps. It seems satisfactory, however, to apply the probe to whatever portion of the lens is accessible, free of the iris. In the presence of a small pupil, the lens may be lifted out by attaching the probe to its centre.

The lens-probe adhesion would appear to provide a securer grip than a capsule forceps, and the risk of capsule rupture to be reduced. However, the use of cryosurgery is dubious in routine cataract extraction where the forceps procedure is perfectly satisfactory and permits simpler instrumentation. Cryosurgery is valuable in dealing with the tense capsule of a swollen lens, or with a subluxated lens, where the danger of further dislocation and loss is reduced because the most gentle approximation of the cold probe to the lens is sufficient to secure adhesion. We have not used this method to enter the vitreous for a dislocated lens, because the retinal probe is too gross and would almost certainly adhere to vitreous structure. A finer probe for intra-vitreal experiment is, however, being developed. It is possible that such an instrument might be useful not only in recovering a dislocated lens, but also in removing intra-ocular foreign bodies.

One caution about cryosurgical lens extraction should be stressed. While a relatively simple device, such as a carbon-dioxide acetone probe, can produce the mild level of sub-freezing required for seizing a cataractous lens, such an instrument is inadequate unless equipped with a warming component so that it can be disengaged if it should become stuck to the iris or the back of the cornea.

\section{Glaucoma}

While Bietti considered the possibility of a cyclocryothermy operation for glaucoma in 1950, he never seemed to become enthusiastic. Polack and deRoetth (1964) have performed cyclocryothermy in 100 cases of glaucoma in the past year, and reported improvement in 25 per cent. Our own work on this disease has been largely confined to animal studies. We were encouraged to try a cyclocryothermy procedure 
as a result of information gained during retinal detachment experiments. We had observed that neuro-epithelium was particularly sensitive to cold, the vascular supply relatively immune, and sclera, muscle, and conjunctiva free from permanent injury. It seemed conceivable that the secretory epithelium of the ciliary body might be damaged without destroying the vascular integrity of the globe, as with extensive diathermy. This was the subject of a separate report by the authors (McLean and Lincoff, 1964) read at a meeting of the American Ophthalmological Society in June, 1964. The report describes experiments to the ciliary body of rabbits, which determined that $-80^{\circ} \mathrm{C}$. applied for $10-15$ seconds was optimal for producing ciliary body destruction. Such applications cause permanent atrophy of the ciliary and pigment epithelium. The low temperature is required because of the thickness and vascularity of the structure. Following such applications, the intra-ocular pressure drops precipitously (Fig. 7), but only temporarily. In 16 to 24 days the eye recovers its normal pressure and aqueous is again produced at apparently normal levels.
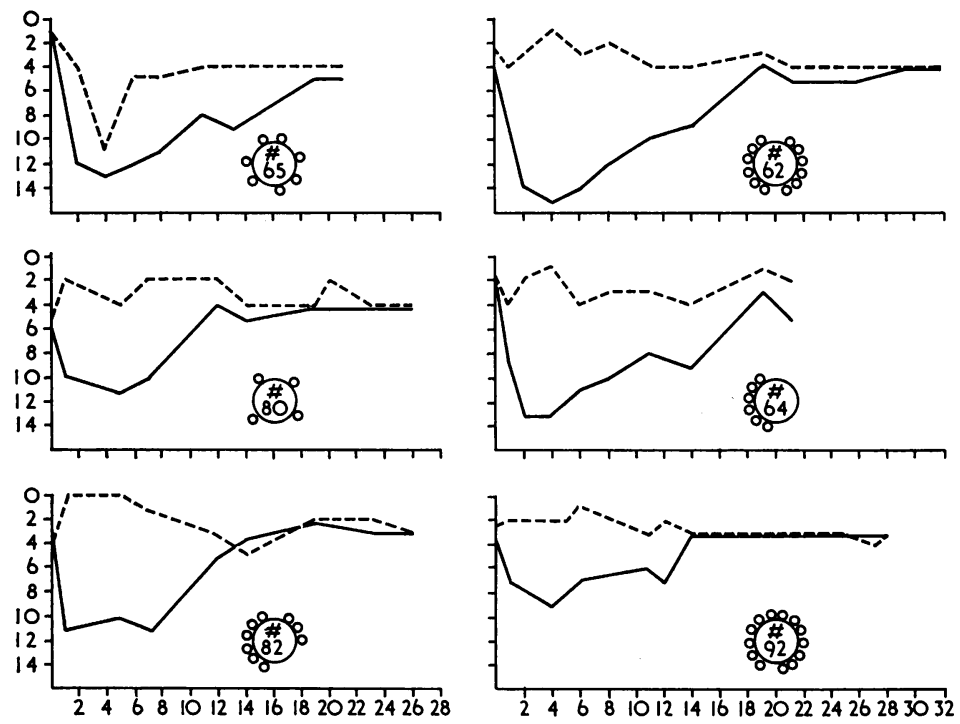

Fig. 7.- Intra-ocular pressure in rabbits following cyclocryothermy at $-80^{\circ} \mathrm{C}$. Ordinate in Schiötz units, abscissa in days.

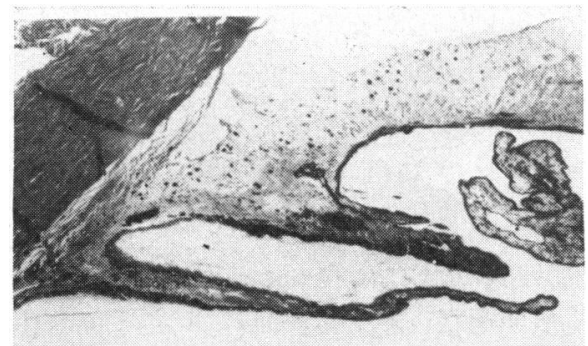

FIG. 8.-Ciliary body of rabbit 30 days after cyclocryothermy at $-80^{\circ} \mathrm{C}$.
Histological examination of these globes shows extensive destruction of ciliary epithelium. Because of the irregular contour of the ciliary villi, however, many of their tips, particularly the more anterior ones beneath the iris (Fig. 8), escape. The amount of survival seems sufficient for restoration of normal function. This is not surprising in the light of the fact that the relatively atrophic ciliary body of chronic glaucoma continues to function sufficiently to maintain glaucomatous pressures. 
More extensive freezing of the ciliary body at $-100^{\circ} \mathrm{C}$. and $-120^{\circ} \mathrm{C}$. has produced permanent lowering of intra-ocular pressure in rabbits (Fig. 9). However, these eyes become phthisical. The problem is to find a therapeutic lesion that will produce a permanent hypotensive effect short of phthisis. After one and a half years of research the solution to this problem is not yet clear.

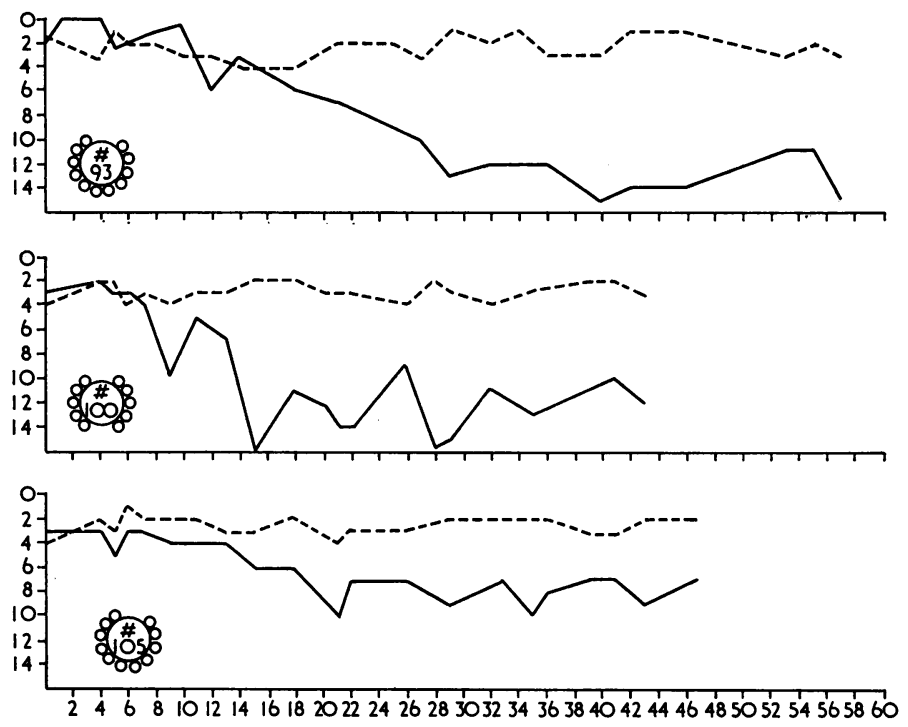

FIG. 9.-Intra-ocular pressure in rabbits after cyclocryothermy at $-120^{\circ} \mathrm{C}$. Ordinate in Schiötz units, abscissa in days.

\section{Tumours}

Our work with regard to tumours has so far been limited. Unfortunately, intraocular tumours in animals are unavailable and attempts to grow the Green melanoma in suitable animals have been unsuccessful. We have frozen three retinoblastomas in two eyes. Two of the tumours were 1 disc diameter in size and the third measured 3 disc diameters. The two smaller tumours were frozen at $-80^{\circ} \mathrm{C}$. and the larger at $-120^{\circ} \mathrm{C}$. The latter temperature was excessive and caused rupture of the choroid and subretınal haemorrhage in and around the tumour. The tumours atrophied in two weeks. Retinal traction developed at the site of the large tumour and caused a falciform detachment of the retina at its nasal periphery. Nearly a year has passed since the freezing treatment, and the tumours in the two eyes have remained inactive. The detachment has not yet shown any tendency to advance. Because retinoblastomas are of neuro-epithelial origin, and this tissue appears to be particularly sensitive to cold, it is possible that cryosurgery will prove of value in their treatment.

We have also treated one case of choroidal metastases of breast carcinoma. Because of the flat nature of this tumour penetration was easier to accomplish. The tumour regressed and the overlying exudative detachment absorbed. We feel that cryosurgery may be of palliative value in the treatment of exudative detachments secondary to metatastic carcinoma, and work on patients with such tumours is to be continued. 


\section{Summary}

Two and a half years of animal experimentation with ophthalmic cryosurgery, and clinical observation of its results in patients, have yielded the following results:

(1) Cryosurgery is superior to diathermy in the treatment of retinal detachment.

(2) Cataractous lenses with tense capsules and subluxated lenses may be removed with greater security by cryopexy than with capsule forceps.

(3) Experiments to date with a cyclocryothermy operation have not been successful in producing a permanent lowering of intra-ocular pressure.

(4) Cryopexy may be useful in the treatment of retinoblastoma and metastatic carcinoma.

\section{REFERENCES}

Bellows, J. G. (1964). Amer. J. Ophthal., 57, 29.

BiETTI, G. (1934). Boll. Oculist., 13, 576. (1950). J. Amer. med. Ass., 142, 889.

COOPER, I. S. (1963). New Engl. J. Med., 268, 743.

Deutschmann, R. (1933). Klin. Mbl. Augenheilk., 91, 450.

Kelman, C. D., and CoOPER, I. S. (1963). Amer. J. Ophthal., 56, 731.

KrWAWICZ, T. (1961). Brit. J. Ophthal., 45, 279. (1963). Ibid., 46, 36.

Lincoff, H. A., McLean, J. M., and Nano, H. (1964). Trans. Amer. Acad. Ophthal. Otolaryng., 68, 412. McLean, J., and Lincoff, H. (1964). Trans. Amer. ophthal. Soc., 62, 385.

Polack, F. M., and DeRoetth, A., Jr. (1964). Invest. Ophthal., 3, 164. 